\title{
Suco clarificado de Caju "Cajuína" - um estudo experimental em tempos de COVID $-19$
}

\section{Clarified cashew juice "Cajuina" - an experimental study}

DOI: $10.46919 / \operatorname{archv1n6-015}$

Recebimento dos originais: 01/09/2020

Aceitação para publicação: 30/10/2020

Lorena de Holanda Gonçalves

Graduanda em Nutrição pelo UNINOVAFAPI

Instituição: Centro Universitário Uninovafapi

Endereço: Rua Vitorino Orthiges Fernandes, 6123 - Uruguai, Teresina - Pi, CEP 64073-505

E-mail: lorenaholanda.21@gmail.com

Luiza Dias Carvalho Santos Brandão

Graduanda em Nutrição pelo UNINOVAFAPI

Instituição: Centro Universitário Uninovafapi

Endereço: Rua Vitorino Orthiges Fernandes, 6123 - Uruguai, Teresina - Pi, CEP 64073-505

E-mail: luizadcsbrandao@gmail.com

\author{
Marielle da Silva Costa \\ Graduanda em Nutrição pelo UNINOVAFAPI \\ Instituição: Centro Universitário Uninovafapi \\ Endereço: Rua Vitorino Orthiges Fernandes, 6123 - Uruguai, Teresina - Pi, CEP 64073-505 \\ E-mail: mariellesilva99@gmail.com \\ Francílio de Carvalho Oliveira \\ Doutor em Engenharia Biomédica pela UNIVERSIDADE BRASIL \\ Instituição: Centro Universitário Uninovafapi \\ Endereço: Rua Vitorino Orthiges Fernandes, 6123 - Uruguai, Teresina - Pi, CEP 64073-505 \\ E-mail: franciliooliveira@ hotmail.com
}

\section{RESUMO}

Objetivo: Realizar a análises do suco clarificado de caju em ambientes não convencionais utilizando materiais alternativos, a partir da observação da obediência às conformidades nas informações rotulares, como o comportamento ácido/básico frente a um indicador natural e a toxicidade da bebida provocada em espécie de Allium cepa. Metodologia: Foram realizadas análises de 14 marcas de cajuína comercializadas em 3 municipios do estado do Piaui, através das informações rotulares, acidez por colorimetria e a toxicidade pelo método de Allium cepa. Resultados: Observou-se que duas das marcas analisadas não estavam em conformidade com a legislação ( $\mathrm{K}$ e L) encontradas no comércio formal de Barras - PI. As amostras avaliadas apresentaram uma variação no grau de acidez com predominância para o meio ácido, sendo 3 amostras ácidas (C e D) adquiridas no comércio de Jatobá do Piauí e (E) adquirida em TeresinaPI e 2 amostras alcalinas (A e B) comercializadas no município de Barras PI. Conclusão: É possível realizar estudos experimentais em ambientes não convencionais deste que cercado do controle das condições ambientais, como temperatura, pressão, umidade etc. Todas as marcas foram consideradas tóxicas. 
Palavras Chaves: Rotulagem, Suco Clarificado, Toxicidade.

\begin{abstract}
Objective: To perform the analysis of clarified cashew juice in unconventional environments using alternative materials, based on the observation of the obedience to conformities in the label information, such as acid / basic behavior in relation to a natural indicator and the toxicity of the drink caused in species of Allium cepa. Methodology: Analyzes of 14 brands of cajuína, which are marketed in 3 cities in the state of Piaui were carried out through the label information, acidity by colorimetry and toxicity by the method of Allium cepa. Results: It was observed that two of the analyzed brands, found in the formal trade of Barras-PI, were not in compliance with the legislation ( $\mathrm{K}$ and $\mathrm{L}$ ). The evaluated samples showed a variation in the degree of acidity with predominance for the acid medium being 3 acid samples $(\mathrm{C}$ and D) acquired in Jatobá do Piauí and (E) acquired in Teresina-PI and 2 alkaline samples (A and B) commercialized in the city of Barras-PI.Conclusion: It is possible to carry out experimental studies in non-conventional environments, since it is surrounded by the control of environmental conditions, such as temperature, pressure, humidity etc. All brands were considered toxic.
\end{abstract}

Keywords: Labeling, Clarified Juice, Toxicity.

\title{
1 INTRODUÇÃO
}

A pandemia causada pela covid-19 que se alastrou pelo mundo trouxe além das irreparáveis perdas de vidas humanas (OMS, 2020), o fechamento, ainda que provisório, dos estabelecimentos de ensino, seja público ou privado, em todas as modalidades. Em Teresina capital do estado do Piauí o cenário não foi diferente, em meados de março de 2020, os decretos municipais e estadual, orientaram a suspensão das atividades de ensino, pesquisa e extensão em toda a esfera educacional (DECRETO ESTADUAL - PIAUI, 2020).

Nesse contexto, nasce à angústia de como dá prosseguimento ao estudo experimental para a conclusão de curso com credibilidade, sem as rotinas laboratoriais necessárias e que foram vivenciadas ao longo do curso de nutrição pelos estudantes. Surgiu, então, a seguinte problemática: Seria possível reproduzir condições experimentais em ambientes diferentes daqueles usualmente utilizados pelos pesquisadores em estudos de cunho experimental com abordagem qualitativa ou quantitativa sem o uso dos laboratórios de análises química, biológica, toxicológica, etc? A nova situação exigiu a reelaboração da hipótese da pesquisa.

Renascendo, assim o referido trabalho de conclusão de curso com os mesmos propósitos do projeto anteriormente idealizado: a análise do suco clarificado de caju - a "cajuína", uma bebida regional oriunda do pseudofruto de uma planta nativa (Anacardium occidentale) das regiões norte e nordeste do Brasil (EMBRAPA, 2009).

A cajuína antes fabricada exclusivamente de forma artesanal, modernizou-se, industrializou-se e conquistou o mercado nacional e internacional (SEBRAE, 2003), trazendo consigo a necessidade de 
obediência às recomendações da legislação vigente no Brasil com o intuito de garantir a seguridade alimentar à população.

Portanto, objetivou-se realizar as análises do suco clarificado de caju em ambientes não convencionais utilizando materiais alternativos, a partir da observação da obediência às conformidades nas informações rotulares, como o comportamento ácido/básico frente a um indicador natural, e a toxicidade da bebida provocada em espécie de Allium cepa.

\section{METODOLOGIA}

\subsection{CENÁRIO DO ESTUDO}

O estudo experimental foi realizado simultaneamente em três municípios do estado do Piauí (Barras, Jatobá do Piaui e a capital Teresina), cidades das respectivas pesquisadoras. Os espaços não convencionais selecionados foram os dormitórios dos pesquisadores.

\subsubsection{Amostra}

Selecionou-se 14 marcas de suco clarificado de caju, no comércio formal do Piauí (Barras, Jatobá do Piaui e a capital Teresina). Essas foram escolhidas de acordo com a disponibilidade na hora da colheita que foram realizadas nos meses de fevereiro a maio de 2020.

\subsubsection{Rotulagem}

Analisou-se 14 rótulos do suco clarificado a partir da leitura direta das informações contidas em suas embalagens. Esses 14 rótulos foram codificados com letras de $\mathrm{A}$ a $\mathrm{N}$ e confrontados com a Resolução da Diretoria Colegiada (RDC) n² 259/2002 e a Instrução Normativa nº. 37/2018.

\subsubsection{Análises experimentais}

Das 14 marcas do suco clarificado obtidos no comércio local, selecionou-se as 5 marcas, sendo 2 fabricadas na cidade de Jatobá do Piauí, 2 no município de Barras e 1 em Teresina (capital do estado do Piauí) para a realização das análises de acidez e toxicidade. A seleção se deu devido ao isolamento social, sendo confortável e seguro para os autores a obtenção dos produtos em locais próximos ao seu domicílio.

\subsubsection{Acidez}

Para a análise da acidez se utilizou o método colorimétrico, utilizando extrato etanólico de beterraba, como indicador; vinagre, como meio ácido; e, detergente a base de amônia, como meio alcalino e as amostras foram codificadas com letras de A a E (Figura 1). 
Figura 1- Ensaio realizado utilizando o extrato etanólico de beterraba como indicador, detergente a base de amônia como meio alcalino e vinagre como meio ácido.

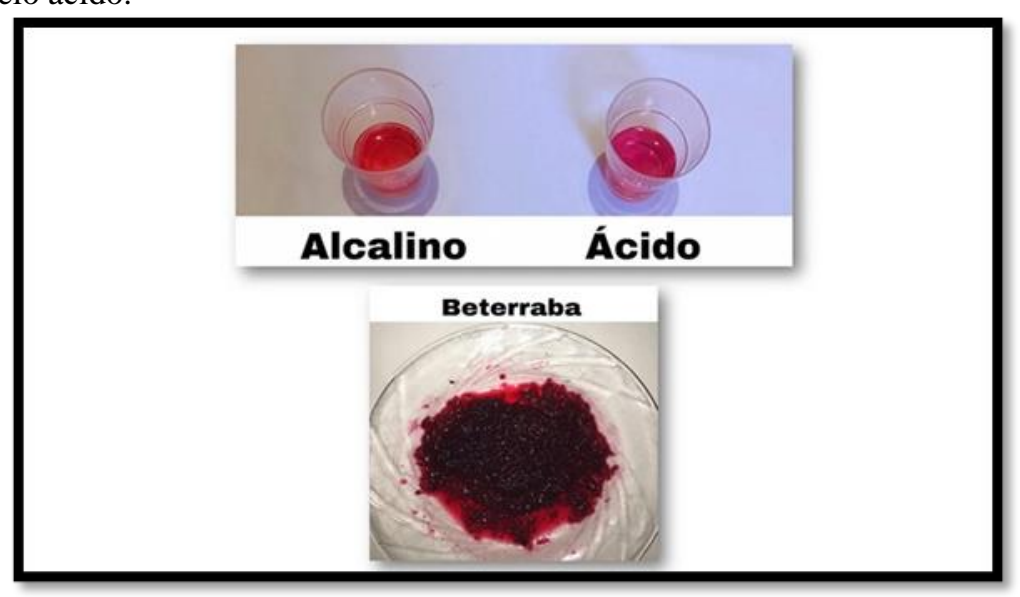

Fonte: Autoria 2020

Foram adicionadas em recipientes de plásticos amostras dos sucos clarificados de caju e acrescentadas a elas 3 gotas da solução indicadora, obtido da infusão de uma colher de sopa de beterraba (Beta vulgaris L.) macerada em $10 \mathrm{~mL}$ de álcool etílico a 70\% (LUCAS et al. 2013). Todas as soluções permaneceram em contato com o indicador por 2 minutos para aferição da coloração.

\subsubsection{Toxicidade}

Os ensaios de toxicidade foram realizados em células vegetais de Allium cepa (obtidos comercialmente) de acordo com o protocolo de Fiskesjo (1985) que se baseia na exposição de bulbos de cebola às concentrações testadas, utilizando cinco repetições para cada. Os bulbos de $A$. cepa foram lavados em água corrente por cerca de 20 minutos, com ajuda de uma lâmina de bisturi cirúrgico se realizou a retirada das raízes secas e camadas externas. Em seguida, foram retirados os parênquimas centrais das coroas de brotamento, fazendo uma pequena incisão em formato de $\mathrm{X}$ para aumentar a absorção das soluções e a uniformidade de brotamento e crescimento e, posteriormente, uma raspagem para retirada de possíveis resíduos. A toxicidade foi avaliada de acordo com a medida do comprimento das raízes, que foram dispostas em local escuro, após a exposição dos bulbos às amostras de cajuína em temperatura ambiente por 72 horas. Para o controle negativo foi utilizado água da torneira, enquanto a solução salina de sulfato de cobre $(0,006 \mu \mathrm{g} / \mathrm{mL})$ foi usada como controle positivo. Para essa etapa foram analisadas 5 marcas de sucos clarificados de caju do mesmo lote e que estavam em temperatura ambiente. De cada marca utilizou-se cinco amostras sendo as mesmas acondicionadas em pequenos copinhos de plástico descartável. A fim de proteger as amostras do excesso de luminosidade se adaptou uma caixa de papelão (Figura 2 D) onde as mesmas foram deixadas até o término do experimento. 
Figura 2- Esquema da toxicidade com água potável utilizando Allium cepa. (A) amostras de cebolas, (B) água mineral, (C) cebolas nos copinhos descartáveis e (D) caixa de papelão.

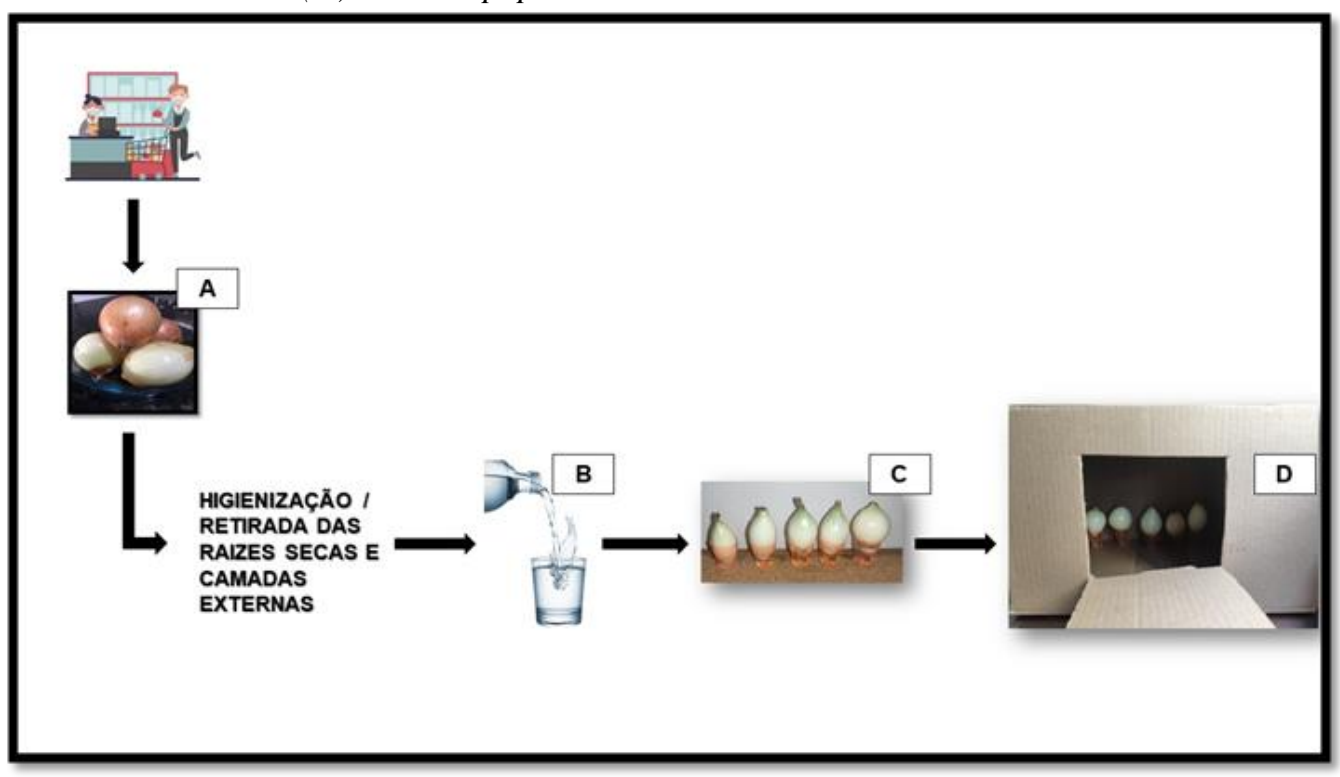

Fonte: Autoria 2020

Realizou-se ainda teste de toxicidade com as águas potáveis (Figura 2) das cidades de Jatobá do Piauí, Barras e de Teresina e também com água mineral de marca Y a fim de verificar o processo de germinação das raízes do Allium cepa, já comprovada na literatura, mas necessárias para a comprovação da eficácia do método frentes as condições que as amostras de cajuína foram submetidas.

Análise estatística

Os resultados obtidos no ensaio de toxicidade foram avaliados com auxílio do Software Graph Pad Prism® 7.0, por análise de variância (ANOVA) de via não paramétrica, com o teste de Newman-Keuls Multiple Comparison Test para múltiplas comparações entre os grupos teste e controle, com níveis de significâncias até $\mathrm{p} \leq 0,05$ e $\mathrm{N}=5$.

Esta pesquisa foi registrada na Coordenação de Pesquisa e Mestrado do Centro Universitário UNINOVAFAPI.

\section{RESULTADO E DISCUSSÃO}

A legislação brasileira (BRASIL, 2018) define o suco de caju clarificado, conhecido como cajuína, como uma bebida não diluída e não fermentada provinda da parte comestível do pseudofruto, obtido por um processo físico de clarificação (Figura 3). 
Figura 3 - Imagem do suco clarificado de caju engarrafado.

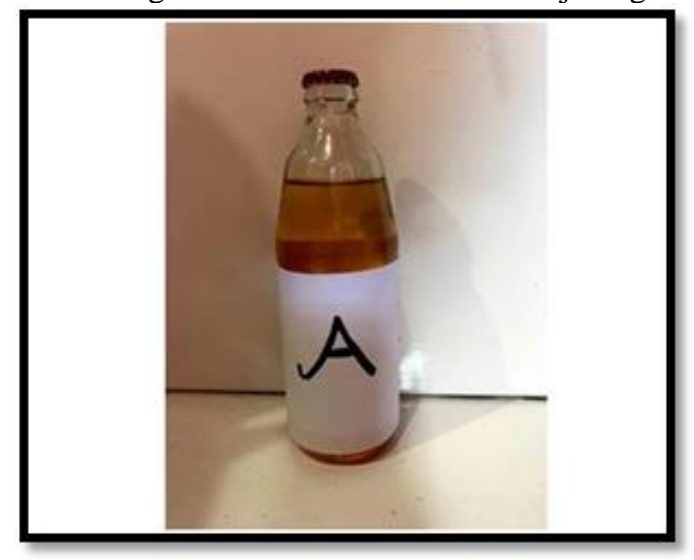

Fonte: Autoria 2020

A bebida deve exibir uma variância da coloração entre o incolor ao amarelo translúcido, sabor característico, levemente ácido e doce, pouca adstringência com aroma específico. A legislação recomenda, no desenvolvimento físico de clarificação, o uso auxiliar de tecnologia autorizada e o produto conquistado deve corresponder sempre aos Padrões de Identidade e Qualidade (PIQ) fixados para suco de fruta (BRASIL, 2018).

Segundo o SEBRAE (2003) a cajuína é um produto totalmente brasileiro cujas principais características são a baixa adstringência e a ausência de turvação. Esta é uma bebida refrescante para ser ingerida de preferência gelada, e muito apreciada nas regiões do Nordeste do país. Além disso, é o suco de caju puro obtido por clarificação, sem adição de açúcar ou conservantes. Após a clarificação o suco é colocado em garrafa de vidro e aquecido em banho-maria, para obter a bebida de sabor e cor âmbar únicos.

\section{ROTULAGEM}

A Resolução da Diretoria Colegiada - RDC N³60/2003 considera que a rotulagem nutricional proporciona ao consumidor conhecer as propriedades nutricionais, como o valor nutricional dos alimentos, contribuindo para um consumo adequado dos mesmos e assim visualizando o que consomem. Segundo Bezerra et al. (2019), o rótulo de um alimento é uma forma de diálogo entre o produto e os consumidores. Com isso, destaca-se a importância de as informações contidas na rotulagem serem precisas para facilitar as escolhas adequadas dos produtos para consumo.

Foi realizado análise dos rótulos de 14 marcas diferentes de suco clarificado de caju "cajuína”, codificadas com as letras de A a $\mathrm{N}$ com a finalidade de identificar se todas as marcas estavam em conformidades. Na Figura 4 é possível visualizar que das 14 marcas analisadas apenas 2 se encontravam em não conformidade codificadas com a letra $\mathrm{K}$ e L encontradas no comércio formal de Barras-PI. As 
conformidades apresentadas são de acordo com a Resolução da Diretoria Colegiada - RDC N²59/2002, na qual foi encontrada nas análises: lista de ingredientes, Identificação da origem, Identificação do lote, prazo de validade e por fim a tabela nutricional.

Figura 4- Análises dos rótulos de sucos clarificados de caju, comercializados no Estado Piauí no ano de 2020.

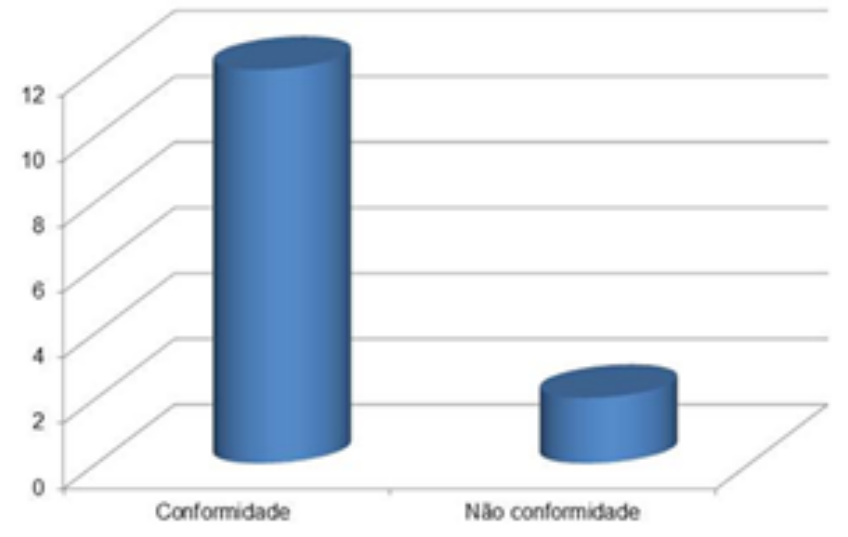

Fonte: Autoria 2020

Como citado acima, somente duas marcas - K e L não atendiam a RDC, já um ponto positivo foi que todas as embalagens analisadas estavam em vidro e, segundo a $\mathrm{RDC}^{\circ}$ 259/2002, a embalagem é destinada para garantir a preservação do produto. As não conformidades apresentadas foram às ausências de informações nos rótulos, na qual não constava a tabela nutricional. Todavia prever a Resolução da Diretoria Colegiada - RDC N³60/2003, que os rótulos devem conter na tabela as seguintes informações: valor energético, carboidratos, proteínas, gorduras totais, gorduras saturadas, gorduras trans, sódio, fibra alimentar, com isso, afirma a Agência nacional de Vigilância Sanitária (ANVISA, 2001) que a informação nutricional presente no rótulo é uma forma de comparar os alimentos e fazer a escolha correta para consumir.

Segundo o Inmetro (2020), o produto em conformidade, oferece para o consumidor de médio a baixo risco à saúde do mesmo e do meio ambiente, por isso a importância dos produtos estarem em conformidades para evitar riscos à saúde do consumidor. Por essa razão é aconselhável ao consumidor que realize sempre a leitura dos rótulos dos produtos, para evitar esses riscos.

\subsection{ACIDEZ}

$\mathrm{Na}$ análise de acidez (Figura 5), observou-se que as amostras codificadas de A a E foram avaliadas e apresentaram variação no grau de acidez com predominância para o meio ácido, sendo 3 amostras ácidas apresentando um tom mais avermelhado identificadas em $\mathrm{C}$ e D, adquiridas no município 
de Jatobá do Piauí; em E, adquirida no comércio de Teresina Piauí e 2 amostras alcalinas em um tom menos avermelhado identificadas em A e B comercializadas no município de Barras Piauí.

Figura 5- Esquema experimental do comportamento ácido/básico das soluções utilizadas como parâmetros.

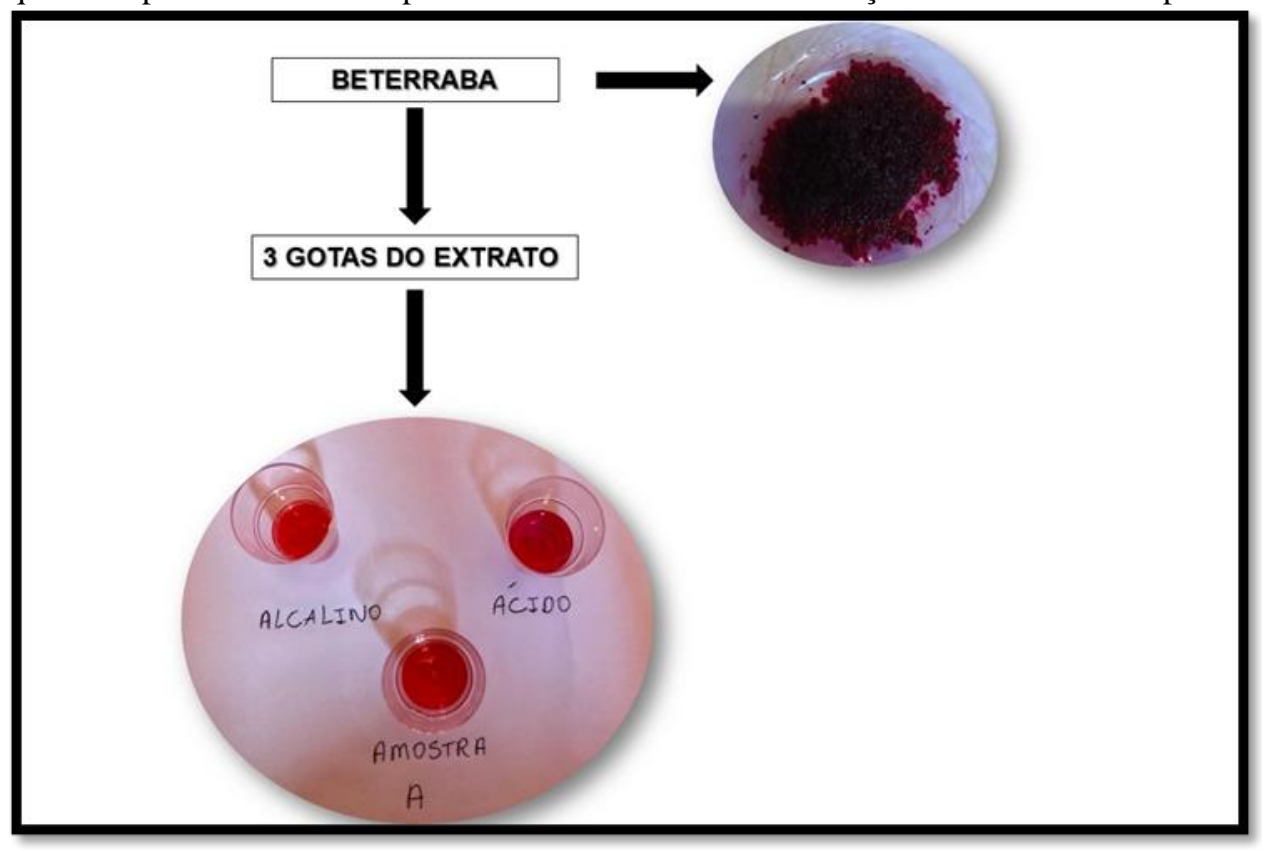

Fonte: Autoria 2020

Segundo Silva et al. (2011) para a indústria de alimentos a acidez é um importante parâmetro de preservação, sendo o alimento ácido indicado para a melhor preservação de alimentos industrializados e que alimentos com baixa acidez são mais propícios à proliferação de microrganismos patogênicos.

Silva et al. (2017) consideram que os valores normais para o pH sanguíneo é de 7,35 a 7,45, faixa fundamental para um bom desempenho de diversas reações no organismo. De acordo com Cruz Neto (2016) é importante que o pH sanguíneo esteja dentro da normalidade para que absorva e conserve na medula óssea os minerais indispensáveis para a saúde e permaneça livre da acidez e complicações ocasionadas pelo excesso de acidez no organismo. Qualquer alimento seja sólido ou líquido como o suco clarificado de caju no qual se apresentou ácido, consumido em excesso que chegue a desestruturar o equilíbrio do pH padrão, mostrando-se prejudicial à saúde do indivíduo. Uma curta variação do $\mathrm{pH}$ no corpo pode deixa o sistema imunológico fragilizado, dando chance para que bactérias, vírus e fungos encontrem ambiente favorável para viver e se reproduzir. (FRANCO, 2008).

Quando há um acúmulo de acidez no corpo, não se consegue absorver direito vitaminas, minerais e suplementos alimentares. Quando o pH do corpo está ácido, o organismo começa a usar os minerais que estão na medula óssea dos ossos do esqueleto pretendo a aumentar o $\mathrm{pH}$ para diminuir a acidez e com isso evitar uma irrigação de células. No entanto quando se ingere frequentemente alimentos muito ácidos, o 
organismo não consegue reduzir essa acidez e o $\mathrm{pH}$ do sangue é diminuído direcionando ácido no transporte de nutrientes e irrigação de células. (YOUNG, 2002)

Sorrentino et al. (2012) demonstraram que o câncer possui certa dificuldade para se desenvolver em um meio alcalino, que possui bastante oxigênio. A ausência de oxigênio desestrutura o sistema imunológico o deixando mais vulnerável e impendido o corpo de agir contra o ataque de células estranhas, ocasionando assim o aparecimento de patologias e alterando o $\mathrm{pH}$ corporal, que se torna ácido, causando ainda mais a carência de oxigênio. Quando o pH se encontra adequado o corpo mantem um nível rico em oxigênio dificultando assim o desenvolvimento de células tumorais.

Segundo Guyton et al. (2011) o equilíbrio ácido/básico é de suma importância também para regular os mecanismos das funções renais. Valores do $\mathrm{pH}$ sanguíneo abaixo do recomendado pode ocasionar acidose, que é causada pelo excesso de íons $\mathrm{H}^{+}$no sangue, sendo a acidose metabólica é consequência da instabilidade causada pelo excesso de ácido não excretado pelo corpo. E no caso de os valores de $\mathrm{pH}$ serem superior ao recomendado podem causar a alcalose que é provocada pela redução de íons $\mathrm{H}^{+}$no sangue, na alcalose metabólica é uma condição oposta, onde há a perdas excessivas de íons $\mathrm{H}^{+}$.

Paula (2007) descreve que os alimentos em que o pH se encontram entre 2,5 e 3,5 como exemplo os sucos e concentrados de frutas citricas, não são quase afetados pela reação de maillard, devido ao seu pH e por serem pobres em compostos aminos. Neste caso o que causa as reações que levam ao escurecimento são a degradação do ácido ascórbico que são catalisadas pelo ácido cítrico e alguns aminoácidos que podem estar presentes. Em um alimento com pH entre 4 e 5, ocorre simultaneamente a reação de Maillard e a degradação do ácido ascórbico. (CHEFTEL et al. 1992)

\subsection{TESTE DE TOXICIDADE}

A Figura 6 ilustra o processo de germinação das raízes de $A$. cepa frente à água potável utilizada como controle negativo à toxicidade e as amostras de sucos clarificados de caju. 
Figura 6- (A) Bulbo de cebolas que desenvolveram raízes durante o período de 72h em água potável, (B) Grupo de cebolas que foram expostas durante $72 \mathrm{~h}$ ao suco clarificado de caju.

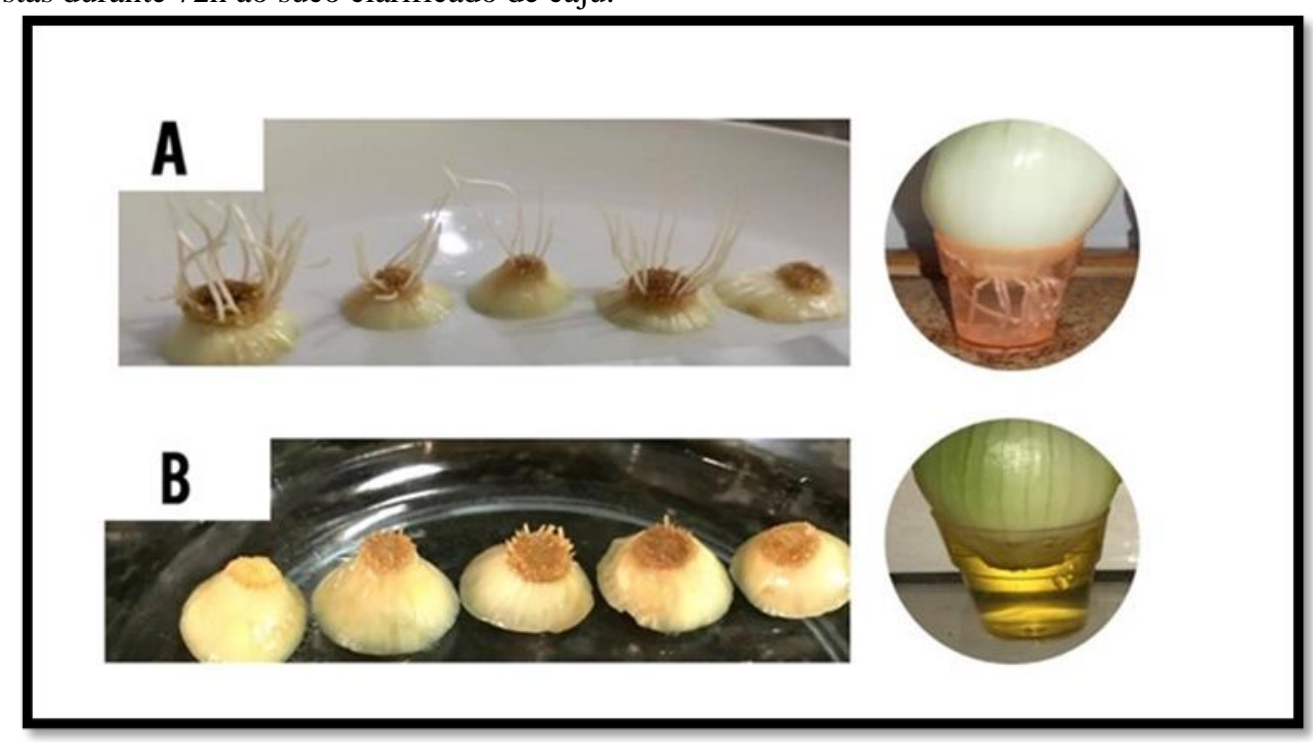

Fonte: Autoria 2020

Pode-se observar que não houve crescimento significativo das raízes do grupo B, comparados com as do grupo A. Contudo, além de não ocorrer o desenvolvimento da germinação foi possível observar aspecto de bulbos desidratados e a inibição do crescimento das raízes na amostra expostas ao suco clarificado de caju.

De acordo com Correa et al.(2016) ao expor um bulbo da cebola à hidratação, o crescimento do tecido meristemático da raiz é estimulado, mas quando exposto ao bulbo na presença de substâncias, a divisão celular tóxica orgânica ou inorgânica de os meristemas da raiz podem ser inibidos, seja atrasando o processo de mitose ou destruição de células. Corroborando com os achados da pesquisa para as amostras de águas das cidades de Jatobá do Piaui, Barras e Teresina, e de água mineral que seguiram o comportamento de não tóxicas.

Arraes (2012) evidencia que os ensaios toxicológicos são realizados com o objetivo de se determinar o efeito nocivo de agentes físicos, químicos e biológicos a diversos organismos avaliando-se assim o potencial de risco à saúde humana e a vida aquática, possibilitando um melhor controle e monitoramento da qualidade dos ecossistemas.

Segundo Akaishi (2003) a compreensão da ação tóxica de alguns compostos pode ser realizada com experimentos laboratoriais conhecidos como bioensaios. O uso de bioensaios em estudo de toxicologia é significativo por permitir estudar os efeitos tóxicos de determinados contaminantes em organismos de forma isolada ou associados, minimizando a influência das variáveis ambientais e possibilitando o estudo da especificidade relativa de alguns efeitos com os contaminantes. 
Roa et al. (2012) em estudo realizado consideraram que esse teste tem sido amplamente utilizado para estudar a toxicidade e genotoxicidade de muitos contaminantes perigosos, como pesticidas, corantes, conservantes alimentares e hidrocarbonetos, onde todos os testes apontaram que A. cepa é mais sensível a detectar toxicidade e genotoxicidade do que outros testes.

A Figura 7 ilustra os resultados da toxicidade das amostras de cajuína, as quais indicaram uma toxicidade em relação ao grupo controle negativo $(\mathrm{SD}=10,77 \pm 1,89)$ em todas as marcas testadas $\mathrm{A}(\mathrm{SD}$ $=2,4 \pm 0,2), \mathrm{B}(\mathrm{SD}=1,2 \pm 0,1), \mathrm{C}(\mathrm{SD}=1,6 \pm 0,2), \mathrm{D}(\mathrm{SD}=1,4 \pm 0,2)$ e $\mathrm{E}(\mathrm{SD}=4,0 \pm 0,8)$ assemelhandose estatisticamente do controle positivo ( $\mathrm{SD}=3,77 \pm 0,79$ ).

Figura 7- Avaliação da toxicidade pela evolução das raízes (em milímetros) após exposição às amostras de bebidas por 72 horas. De A-E refere-se as cinco marcas utilizada. As diferenças entre os grupos foram estabelecidas por Análise de Variância (ANOVA), seguido de t-Student-Neuman-Keuls comparado com o grupo controle negativo (CN) e controle positivo (CP). * indica uma diferença estatisticamente significativa.

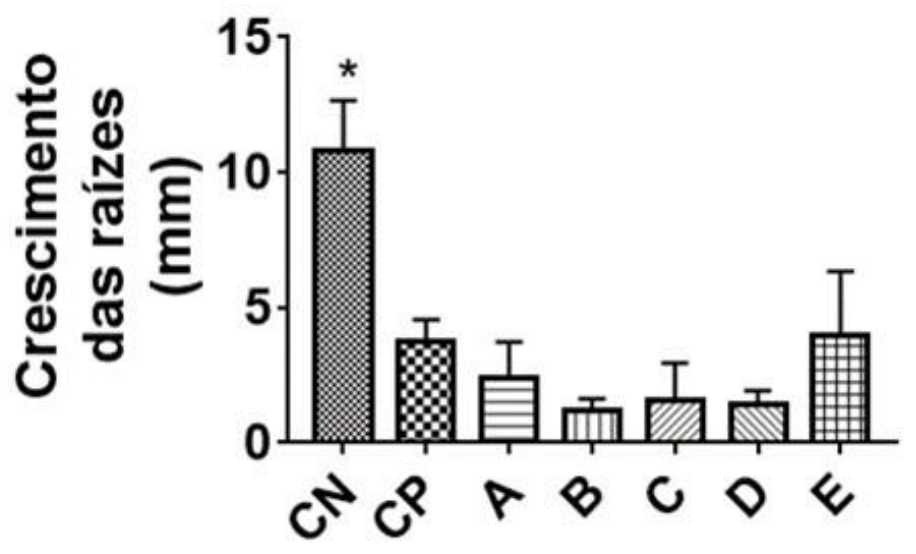

Fonte: Autoria 2020

Com base nos resultados apresentados foi possível observar uma diferença estaticamente significativa, no qual os valores encontrados das germinações dos bulbos de cebolas das marcas analisadas se aproximaram ao valor encontrando no controle positivo, podendo considerar o teste de toxicidade positivo para o suco clarificado de caju.

Brião et al. (2011) evidenciaram que em consequência do tratamento térmico e armazenamento prolongados, decorre a produção de reações de escurecimento não enzimático, sendo representado pelas reações de caramelização, reação de Maillard e oxidação do ácido ascórbico (vitamina C). O processo de tratamento térmico é uma das etapas mais críticas na fabricação do suco clarificado, sendo utilizado para a conservação do produto, mas é responsável pelas modificações das suas propriedades físico-químicas.

Apesar de em certas condições essas reações produzirem sabor agradável, aroma e coloração, podem também gerar coloração e sabor indesejáveis e alterar a qualidade do alimento durante o processamento e armazenamento. Um dos pontos negativos mais evidentes do escurecimento dos 
alimentos e dos produtos formados a partir dele corresponde à destruição ou redução do valor nutricional de aminoácidos essenciais como arginina, lisina, metionina e triptofano, além de redução da digestibilidade do alimento (ARAUJO, 1999; MEHTA; DEETH, 2016). Muitos fatores influenciam a velocidade desta reação, sendo a temperatura, o tempo, a atividade da água, $\mathrm{pH}$, o tipo e proporção de açúcares redutores, os compostos amino e a fonte de reagente (KWAK et al., 2005; MATMAROH et al., 2006).

Estudos mostraram que a decomposição do ácido ascórbico resulta na produção de furfural e 5 hidroximetilfurfural (Huelin, 1953; Huelin et al, 1971; Kanner, et al, 1981; Robertson e Samaniego,1986). Existem também evidências de que a produção de 5- HMF e furfural seja causada em decorrência da desidratação ácido-catalítica de açucares (CONSIDINE; CONSIDINE, 1982; ROIG et al.,1994; WEDZICHA, 1984).

De acordo com Nascimento et al., (2003), o conhecimento da dinâmica do processo térmico é fundamental no controle da coloração âmbar típica da cajuína, pois se sabe que o tempo e a temperatura influenciam efetivamente na concentração de ácido ascórbico, açucares, aldeídos furânicos e outros compostos constituintes dos produtos alimentícios. Sendo esses compostos responsáveis pelas reações de escurecimento não-enzimático ocorridos no decorrer desse estagio, além da formação de compostos indesejáveis como a 5-hidroxilmetilfurfural.

Segundo Morales (2009), o HMF é considerado tóxico e prejudicial tanto para saúde humana quanto das abelhas. Em humanos, altas concentrações de HMF podem apresentar atividade citotóxica, provocando irritação dos olhos, trato respiratório superior, pele e membranas mucosas, além de apresentar potencial genotóxico.

O estudo realizado por Pires (2013), a partir da investigação da toxicidade do Hydroxymethylfurfural e Thiamethoxam para Apis mellífera L., concluiu que ambos foram tóxicos para A. mellífera, independente da forma de exposição.

Pelo fato do suco clarificado não ser diluído, e ter todo um cuidado para não ocorrer à destruição da Vitamina $\mathrm{C}$ durante o tratamento térmico, a mesma acaba sendo encontrada de forma integral ao final do produto. A partir disso, pode se considerar que a não padronização da temperatura no processo do tratamento térmico é a principal causa que influencia a formação do escurecimento não enzimático consequente da reação de Maillard, produção do 5- Hidroximetilfurfural, que é um marcador intermediário da RM e a oxidação do ácido ascórbico, tornando-se assim a toxicidade no suco clarificado de caju.

Sabe-se que os Produtos da Reação de Maillard correspondem a um grupo heterogêneo de substâncias de baixo peso molecular. O HMF é produzido rapidamente durante o processamento térmico 
e no armazenamento prolongado, principalmente em alimentos ricos em carboidratos e lipídeos (RAJCHL et al., 2013; ROSATELLA et al., 2011). Corroborando com os achados da pesquisa, em estudo realizado por Damasceno (2007), os resultados mostraram que a temperatura do tratamento térmico é o principal fator que influencia no escurecimento e formação do 5-HMF.

\section{CONCLUSÃO}

Portanto conclui-se que é possível realizar estudos experimentais em ambientes não convencionais desde que cercado do controle das condições ambientais, como temperatura, pressão, umidade etc., e que o produto analisado apresenta informações rotulares condizentes com as recomendações da legislação brasileira, mas que há muito ainda a ser estudado acerca de sua produção, concentração dos produtos resultantes desse processo, bem como da quantidade máxima diária a ser recomendada pelos profissionais de nutrição.

\section{REFERÊNCIAS}

AGÊNCIA NACIONAL DE VIGILÂNCIA SANITÁRIA. Rotulagem nutricional obrigatória: manual de orientação aos consumidores Educação para o Consumo Saudável. Universidade de Brasília Departamento de Nutrição: Ministério da Saúde, 2001.

AKAISHI, F. M. Aplicação de biomarcadores de contaminação ambiental em estudos de laboratório e monitoramento em campo. 2003. 123f. Dissertação (Mestrado) - Curso de Ciências Biológicas, Universidade Federal do Paraná, Curitiba, 2003.

ARAÚJO, J.M.A. Química de Alimentos: teoria e prática. 2. Ed. Viçosa: UFV, 1999.

ARRAES, A. I. O. M. E; LONGHIN, S. R. Otimização de ensaio de toxicidade utilizando o bioindicador allium cepa como organismo teste. Enciclopédia Biosfera, Centro Científico Conhecer, Goiânia, v. 8, n. 14, jun. 2012.

BEZERRA, M. Q. M; MELO, F. I. M. L; SILVA, L. E. M. Alimentos Transgênicos e a Integralidade da Proteção ao Consumidor: A Importância Da Rotulagem. Actio Revista de Estudos Jurídicos, n. 28, v. 2, jul./dez. 2019.

BRASIL, Ministério da Agricultura, Pecuária e Abastecimento. Instrução Normativa no 37 de 1 de outubro de 2018. Art. $1^{\circ}$ - Aprovar o Regulamento técnico geral para fixação dos padrões de identidade e qualidade para polpa de fruta conforme consta do Anexo I. Brasília, DF, 2018.

BRASIL. Agência Nacional de Vigilância Sanitária. Regulamento técnico para rotulagem de alimentos embalados. RDC 259, 20 de setembro de 2002. Diário Oficial da União. 23 set. 2002. (184): 33; Seção 1 . 
BRASIL. Agência Nacional de Vigilância Sanitária. Regulamento técnico sobre rotulagem nutricional de alimentos embalados. RDC 360, 23 de dezembro de 2003. Diário Oficial da União. 26 dez. 2003. (251):33; Seção 1.

BRIÃO, V. B. et al. Cinética do escurecimento não-enzimático com soluções modelo de açúcares e aminoácidos em pH neutro e ácido. Acta Scientiarum. Technology, Maringá, v. 33, n. 1, p. 87-93, 14 mar. 2011.

CHEFTEL, J. C.; CHEFTEL, H. Introducción a la bioquímica y tecnología de los alimentos. 2. ed. Zaragoza: Editorial Acríbia, 1992. V. 1.

CONSIDINE, D. M.; CONSIDINE, G. D. In. Foods and food production encyclopedia. New York: Van Nostrand Reinhold Co, 1982.

CORREA, M. N.; V. Ventura \& T. da Silva. 2016. Cytotoxic, genotoxic and mutagenic effects of sewage sludge on Allium cepa.Chemosphere, 148: 481- 486.

CRUZ NETO, B. F. Benefícios da água com pH Alcalino: Saúde ou doença, você decide. Educação, Tecnologia e Cultura - ETC., [S.1.], n. 14, jun. 2016. ISSN 2525-3859. Disponível em: http://www.publicacoes.ifba.edu.br/index.php/etc/article/view/8. Acesso em: 11 out. 2020.

DAMASCENO, L. F. Estudo das interações polifenol-proteína e das reações de escurecimento nãoenzimático para o processamento de cajuína. 2007. 87 f. Dissertação (Mestrado em Pesquisa e Desenvolvimento de Tecnologias Regionais) - Universidade Federal do Rio Grande do Norte, Natal, 2007.

DECLARAÇÃO do Fornecedor. Disponível em: http://www.inmetro.gov.br/qualidade/declaFornecedor.asp. Acesso em: 11 out. 2020.

FISKESJÖ, G. The Allium test as a standard in environmental monitoring. Hereditas, Lund- Sweden, v. 102, n. 1, p. 99-112, 1985.

FRANCO, B. D. G. M., Landgraf, M. Microbiologia dos alimentos. São Paulo: Editora Atheneu, 2008.

GUYTON, A. C.; HALL, J. E. Tratado de fisiologia médica. Rio de Janeiro: Elsevier, 2011.

HUELIN, F. E.; COGGIOLA, I. M.; SIDHU, G. S.; KENNETT, H. The anaerobic decomposition of ascorbic acid in the $\mathrm{pH}$ range of foods and in more acid solutions. Journal of The Science of Food and Agriculture, [S.1.], v. 22, n. 10, p. 540-542, out. 1971.

HUELIN, F.E. Volatile products of apples (III) identification of aldehydes and ketones. Food Research, 1953.

KANNER, J.; HAREL, S.; FISHBEIN, Y.; SHALOM, P. Furfural accumulation in stored orange juice concentrates. Journal df Agricultural and Food Chemistry, [S.L.], v. 29, n. 5, p. 948-949, set. 1981.

KWAK, E. J.; LEE, Y. S.; MURATA, M.; HOMMA, S.Effect of pH control on the intermediates and melanoidins of nonenzymatic browning reaction. LWT - Food Science and Technology, 38, 2005. 
LUCAS, Mônica et al. Indicador natural como material instrucional para o ensino de química. Experiências em Ensino de Ciências, Blumenau, v. 8, n. 1, abr. 2013.

MARTINS, M. N. C.; SOUZA, V. V.; SOUZA, T. S. Cytotoxic, genotoxic and mutagenic effects of sewage sludge on Allium cepa. Chemosphere, Alegre, v. 148, p. 481-486, abr. 2016.

MATMAROH, K.; BENJAKUL, S.; TANAKA, M. Effect of reactant concentrations on the Maillard reaction in a fructose-glycine model system and the inhibition of black tiger shrimp polyphenoloxidase. Food Chemistry, 98, 2006.

MEHTA, B. M.; DEETH, H. C. Blocked Lysine in Dairy Products: Formation, Occurrence, Analysis, and Nutritional Implications. Comprehensive Reviews in Food Science and Food Safety, v. 15, 2016.

MORALES, F.J. Hydroxymethylfurfural (HMF) and related compounds. In: STADLER, R.H.; LINEBACK, D.R. Process-induced food toxicants occurrence, formation, mitigation, and health risks. Hoboken: Stadler \& Lineback, 2009.

NASCIMENTO, R. F.; AQUINO, F. W. B.; AMORIM, A. G. N.; PRATA, L. F. Avaliação do tratamento térmico na composição química e na qualidade da cajuína. Ciência e Tecnologia de Alimentos, [S.1.], v. 23, n. 2, p. 217-221, ago. 2003.

ORGANIZAÇÃO MUNDIAL DA SAÚDE (OMS). Folha informativa COVID-19 - Escritório da OPAS e da OMS no Brasil. Disponível em: https://www.paho.org/pt/covid19. Acesso em: 05 out. 2020.

PAULA, G. A. de. Caracterização físico-quimica e estudo do escurecimento enzimático em produtos derivados de açaí (Euterpe oleraceae Mart). 2007. 91 p. Dissertação (Mestrado em Tecnologia de Alimentos) Universidade Federal do Ceará, Fortaleza.

PIAUÍ, Governo do Estado. Decretos Estaduais - Novo Coronavírus. Disponível em: https://www.pi.gov.br/decretos-estaduais-novo-coronavirus/. Acesso em: 05 out. 2020.

PIRES, E. P. Toxicidade do hidroxymetilfurfural e thiametoxam para Apis mellifera Linnaeus, 1758 (Hymenoptera: Apidae). 2013. 57 p. Dissertação (Mestrado em Agronomia/Entomologia) Universidade Federal de Lavras, Lavras, 2013.

RAIMUNDO, M. S. N.; FERNANDO, A. P. A.; FRANCISCO, F. A. P. Processamento do pedúnculo de caju: cajuína. Fortaleza: Embrapa Agroindústria Tropical, 2009.

RAJCHL, A. et al. M. Rapid determination of 5-hydroxymethylfurfural by DART ionization with timeof-flight mass spectrometry. Analytical and Bioanalytical Chemistry, n. 405, 2013.

ROA, O.; YEBER, Mc.; VENEGAS, W.. Genotoxicity and toxicity evaluations of ECF cellulose bleaching effluents using the Allium cepa L. Test. Brazilian Journal Of Biology, [S.1.], v. 72, n. 3, p. 471-477, ago. 2012.

ROBERTSON, G. L.; SAMANIEGO, C.M.L. Effect of Initial Dissolved Oxygen Levels on the Degradation of Ascorbic Acid and the Browning of Lemon Juice during Storage. Journal of Food Science, [S.1.], v. 51, n. 1, p. 184-187, jan. 1986. 
ROIG, M. G.; BELLO, J.F.; RIVERA, Z.S.; KENNEDY, J.F. Possible additives for extension of shelflife of single - strength reconstituted citrus juice aseptically packaged in laminated cartons. International Journal of Food Sciences and Nutrition, 1994.

ROSATELLA, A. A. et al. 5-Hydroxymethylfurfural (HMF) as a building block platform: Biological properties, synthesis and synthetic applications. Green Chemistry, v. 13, 2011.

SILVA, M. L. T, et al. Alterações do Equilíbrio Acidobásico. In: WAITZBERG, D. L. Nutrição Oral, Enteral e Parenteral na Prática Clínica. 5 ed. Rio de Janeiro: Atheneu; 2017.

SILVA NETO, R. M.; ABREU, F. A. P.; PAIVA, F. F. A. Cajuína: como produzir com qualidade. Teresina/PI: Edição SEBRAE, 2003.

SILVA, L. R.; BARRETO, N. D. S.; BATISTA, P. F.; ARAÚJO, F. A. R.; MORAIS, P. L. D. Caracterização de frutos de cinco acessos de juazeiro (Zizyphus joazeiro Mart.). Revista Brasileira de Produtos Agroindustriais, Campina Grande, v.13, n.1, p.15-20, 2011.

STADLER, Richard H.; LINEBACK, David R. Process-Induced Food Toxicants: Occurrence, Formation, Mitigation, and Health Risks. [S.1.]: John Wiley \& Sons, 2008.

WARGURG, O. H.. apud SORRENTINO, V. Enzima e oxigenação celular e acidose celular como causa de câncer. Revista de pesquisa SCIENCE, v.137, n. 3523, julho. 1962. Article Scientific, UFRS, 2012.

WEDZICHA, B. L. Chemistry of Sulphur dioxide in foods. Essex, UK: Elsevier Applied Science Publishers Ltda, 1984.

YOUNG, R. O. The pH miracle. 5 ed. New York: Editora New York, NY- Warner Books, 2002.

\section{ANEXO 1}

\section{REGRAS SUBMISSÃO REVISTA - ARCHIVES OF HEALTH}

$\mathrm{O}$ AH aceita apenas artigos originais, não publicados em outras revistas. Aceitamos artigos apresentados em eventos, desde que a informação seja disponibilizada pelos autores.

Os padrões de formatação e preparação de originais são:

- Máximo de 20 páginas;

- Idiomas permitidos: português, inglês e espanhol;

- Autoria: máximo de 8 autores por artigo;

- Fonte Times New Roman tamanho 12, espaçamento entre linhas 1,5; 
- Figuras, Tabelas e Pinturas devem aparecer junto com o texto, editáveis, em fonte 10, tanto para o conteúdo quanto para o título (que deve vir logo acima dos elementos gráficos) e fonte (que deve vir logo abaixo do elemento gráfico).

- Título em português e inglês, no início do arquivo, com fonte 14;

- Resumo e abstract, juntamente com palavras-chave e palavras-chave, com espaçamento simples, logo abaixo do título;

- O arquivo submetido não deve conter a identificação dos autores.

\section{ANEXO 2}

REVISÃO ORTOGRÁFICA

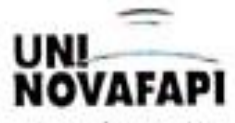

CURSO: NUTRIÇÃO

DISCIPLINA: TRABALHO DE CONCLUSÃO DE CURSO (8". Série)

PROFA.: NORMA SUELI ALBERTO

\section{DECLARAÇ̄̃o DE REVISÃo ORTOGRÁFICA}

Declaro para os devidos fins que eu, Clézia Maria Coutinho Bento, CPF:274.275.603-53, licenciada em Biologia (UFP) e em Pedagogia pela (Faculdade IESM), realizei a REVISĀO ORTOGRÁFICA do Trabalho de Conclusằ de Curso de Nutriçăo intitulado Suco Clarificado de Caju "Cajuína"- Um Estudo Experimental em Tempos de COVID-19 de autoria de Lorena de Holanda Gonçalves, Luiza Dias Carvalho Santos Brandão, Marielle da Silva Costa e orientado pelo Professor Dr. Francilio de Carvalho Oliveira, do Centro Universitário Uninovafapi, no periodo letivo 2020.2 .

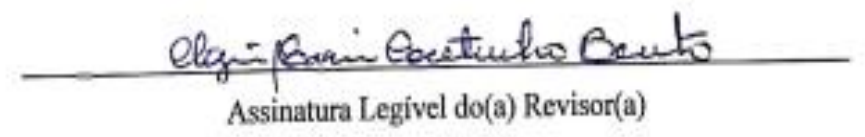

Data: $281 \mathrm{d1} / 2000$ 


\title{
ANEXO 3
}

TRADUÇÃO INGLÊS

\author{
DECLARAÇÃo DE TRADUÇÃO
}

Declaro para os devidos fins que eu, Duilio Rodrigues de Santana, CPF-259.806.10391, licenciado em Letras/nglês pela Universidade Estadual do Piaui, realizei a TRADUÇĀO para a língua inglesa do resumo do Trabalho de Conclusão de Curso de Nutrição intitulado Suco Clarificado de Caju "Cajuina"- Um Estudo Experimental em Tempos de COVID-19, de autoria de Lorena de Holanda Goncalves, Luiza Dias Carvalho Santos Brandão e Marielle da Silva Costa, e orientado pelo Professor Dr. Francilio de Carvalho Oliveira, do Centro Universitánio Uninovafapi, no periodo letivo 20202.

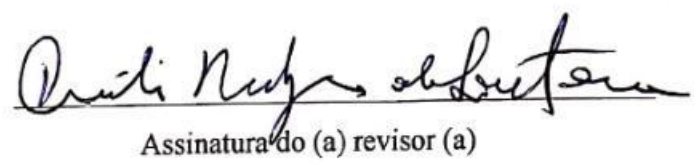

Data: $28,11,2020$ 


\section{ANEXO 4}

APROVAÇÃO COORDENAÇÃO DE PESQUISA E MESTRADO

\section{UNan \\ NOVAFAP

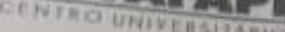

\section{TERMO DE CONSENTIMENTO DA INSTITUICĀO}

Eu, RTTA DE CÁSSLA SÉrVIO MENDES LOPES, Diretora de Ensino do Centro Universitário UNINOVAFAPL, declaro que as alunas LORENA DE HOLANDA GONCALVES, LUIZA DIAS CARVALHO SANTOS BRANDẢO E MARIELLE DA SILVA estĩo autorizadas a realizar nesta Instituiç̧ı o projeto de pesquisa: "INVESTIGAÇẢO DA TOXICIDADE E PROPRIEDADES FISICO-QUIMICAS DO SUCO CLARIFICADO EM CAJU "CAJUINA", sob a responsabilidade do

Professor Dr. Francilio de Carvalho Oliveira.

Os custos financeiros para o desenvolvimento da pesquisa serão de responsabilidade dos

Os alunos deveräo estar cientes que só poderäo utilizar os laboratórios nos horários das atividades acadêmicas e com o conhecimento do coordenador do curso envolvido, isto é, no horário da oferta da atividade académica da disciplina e sempre acompanhados pelo professor orientador.

Informo-the ainda, que a pesquisa somente será iniciada após a aprovaçẫo da Coordenação de Ensino do Centro Universitário Uninovafapi, para garantir a todos os envolvidos os referenciais básicos da ética, isto é, autonomia, nåo maleficência, benevolência e justiça.

Teresina, 09 de Dezembro de 2019

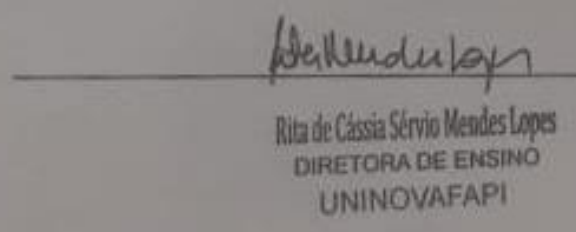

